\title{
Políticas públicas e educação em saúde nos projetos de extensão na universidade
}

\author{
Public policies and health education in extension projects at the university \\ Políticas públicas y educación sanitaria en proyectos de extensión universitaria
}

\section{Resumo}

Objetivo: Caracterizar os projetos de extensão dos cursos da área da saúde de uma universidade pública da Região Norte do Brasil, estabelecendo relações entre políticas públicas e educação em saúde. Metodologia: Pesquisa exploratória, descritiva de abordagem qualitativa com análise de conteúdo de Bardin. Foram analisados os relatórios finais das Ações Curriculares de Extensão (ACE) dos cursos de Educação Física, Enfermagem, Farmácia, Fisioterapia, Medicina e Odontologia, executados nos anos de 2016 e 2017, mediante a utilização de um instrumento contendo questões relacionadas à caracterização do projeto, atividades que contemplassem políticas públicas e educação em saúde. Resultados: Os relatos da comunidade atendida foram elencados em categorias que melhor representaram os impactos das ações de extensão relacionadas às políticas públicas e educação em saúde. A extensão foi apontada como ferramenta para transformar hábitos na comunidade e aperfeiçoar o aprendizado discente. Conclusão: As ações contemplaram políticas públicas de saúde por meio da educação em saúde com alcance na comunidade. Contudo, é necessário promover ações de extensão às populações em situação de vulnerabilidade com um alcance maior dos grupos sociais.

Palavras-chave: Política pública; Educação em saúde; Relações comunidade-instituição; Universidades; Mudança social; Ensino.

\footnotetext{
Abstract

Objective: To characterize Health Extension Projects at a university in the northern region of Brazil, establishing relations between Public Policies and Health Education. Methods: Exploratory descriptive research with a qualitative approach with Bardin Content Analysis. The final reports of the Curricular Extension Actions (ACE) of the Physical Education, Nursing, Pharmacy, Physiotherapy, Medicine and Dentistry courses, performed in the years 2016 and 2017, were
} 
Research, Society and Development, v. 10, n. 4, e58010414379, 2021

(CC BY 4.0) | ISSN 2525-3409 | DOI: http://dx.doi.org/10.33448/rsd-v10i4.14379

analyzed, using an instrument containing questions related to the characterization of the project, activities that included Public Policies and Health Education. Results: The speeches of the community served were listed in categories that best represented the impacts of extension actions related to Public Policies and Health Education. Extension was pointed out as a tool to transform habits in the community and improve student learning. Conclusion: The actions contemplated the implementation of public health policies through health education with reach in the community. However, it is necessary to promote health to populations in situations of vulnerability with a wider reach of social groups.

Keywords: Public policy; Health education; Community-institutional relations; Universities; Social change; Teaching.

\section{Resumen}

Objetivo: Caracterizar los Proyectos de Extensión en Salud en una universidad del Norte de Brasil, estableciendo relaciones entre Políticas Públicas y Educación en Salud. Metodología: Investigación descriptiva exploratoria con enfoque cualitativo con Bardin Content Analysis. Se analizaron los informes finales de las Acciones de Extensión Curricular (ACE) de los cursos de Educación Física, Enfermería, Farmacia, Fisioterapia, Medicina y Odontología, realizados en los años 2016 y 2017, utilizando un instrumento que contiene preguntas relacionadas con la caracterización del proyecto, actividades que contemplaron Políticas Públicas y Educación para la Salud. Resultados: Los discursos de la comunidad atendida fueron listados en las categorías que mejor representaron los impactos de las acciones de extensión relacionadas con Políticas Públicas y Educación para la Salud, la extensión fue señalada como una herramienta para transformar hábitos en la comunidad y mejorar el aprendizaje de los estudiantes. Conclusión: Las acciones incluyeron la implementación de políticas públicas de salud a través de la educación en salud con extensión a la comunidad. Sin embargo, es necesario promover la salud de poblaciones vulnerables con una gama más amplia de grupos sociales.

Palabras clave: Políticas públicas; Educación para la salud; Relaciones comunidad-institucionales; Universidades; Cambio social; Ensenãnza.

\section{Introdução}

A universidade é um ambiente de formação multidimensional, ancorada em três pilares: ensino, pesquisa e extensão. Através da interação entre alunos, professores e programas institucionais, a extensão universitária promove ações na comunidade. Entre os benefícios adquiridos com essa prática, destaca-se o desenvolvimento de habilidades de comunicação dos discentes com a comunidade, a produção científica a partir de inquietações que surgem com as práticas e o impacto para a saúde da população. De acordo com Silva, Ribeiro e Júnior (2013), o processo formativo em saúde desenvolvido com base em ações extensionistas articula ensino e pesquisa, produz novas práticas de cuidado e formação integral por meio da aprendizagem técnica, ética, responsabilidade cidadã e compromisso social.

A extensão universitária possui grande relevância para a formação acadêmica, uma vez que permite a vivência em diversos cenários sociais e ainda agrega aprendizado teórico-prático à formação discente. Conforme Sousa, Carneiro e Rocha (2017), a extensão universitária é caracterizada como uma dimensão da universidade que está pautada em diversas referências epistemológicas e metodológicas. A partir disso, está configurada por meio de diversas formas de pensamento, cumprindo papéis que vão para além do âmbito da formação, bem como as relações com outros setores da sociedade através da socialização dos saberes.

Biscarde, Pereira-Santos e Silva (2014) referem que o reconhecimento da cidadania é fundamental no enfrentamento da realidade socioeconômica e sanitária, ressaltam ainda a necessidade de constante reflexão acerca da formação em saúde. Além disso, admitem que a formação deva ir para além de habilidades técnicas para promover mudanças consistentes nos fatores condicionantes e determinantes da saúde, bem como contribuir para a sustentação dos princípios do Sistema Único de Saúde (SUS).

Conforme Medeiros (2017), no final dos anos 1970 e início dos anos 80 ocorreu a introdução de novos espaços para o debate político, filosófico e social no Brasil. Isso foi possível a partir do processo de abertura política que culminou com o fim da ditadura e com o avanço da redemocratização, promovendo a ideia de que a universidade tem um papel primordial no retorno à democracia. Nesse sentido, destaca-se o Programa de Extensão da Universidade (PROEXT), criado em 2003 com o objetivo de apoiar Instituições Públicas de Ensino Superior no desenvolvimento de programas e projetos de extensão com vistas ao 
Research, Society and Development, v. 10, n. 4, e58010414379, 2021

(CC BY 4.0) | ISSN 2525-3409 | DOI: http://dx.doi.org/10.33448/rsd-v10i4.14379

desenvolvimento de políticas públicas no âmbito universitário.

Biscarde, Pereira-Santos e Silva (2014), afirmam que o Projeto Político Pedagógico de curso deve contemplar não somente o conhecimento técnico-científico, mas possuir um compromisso com o aspecto ético-político relacionado à cidadania e também com a emancipação dos sujeitos e coletividades desse contexto, oferecendo ao futuro profissional a oportunidade de conhecer e intervir na realidade, e contribuir para o seu desenvolvimento a partir de uma perspectiva crítico-reflexiva, valorizando a interdisciplinaridade e aspectos humanísticos, voltados às necessidades quanto à saúde da população.

As atividades de extensão universitária possuem papel primordial na melhoria da qualidade de vida de uma comunidade, sendo a educação em saúde uma ferramenta capaz de transformar realidades e beneficiar a comunidade por meio da apreensão dos conteúdos trabalhados. Para Salci (2013), a educação em saúde é uma ferramenta importante para a promoção da saúde, o envolvimento dos aspectos teóricos e filosóficos deve orientar a prática dos profissionais da área da saúde, com vistas ao desenvolvimento de um trabalho direcionado aos princípios da promoção da saúde.

Para promover a saúde por meio da educação em saúde é importante ter visão holística, valorizar a conscientização sobre os direitos e deveres no atendimento à saúde, desenvolver competências como a socialização, buscar o envolvimento por meio das relações interpessoais e promover a educação para a cidadania.

Nesse sentido, Capella (2018) afirma que as políticas públicas podem ser definidas como a totalidade de ações que os governos nacionais, estaduais ou municipais traçam para alcançar o bem-estar do interesse público. Portanto, esse estudo objetivou caracterizar os projetos de extensão dos cursos da área da saúde de uma universidade pública da Região Norte do Brasil, estabelecendo relações entre políticas públicas e educação em saúde.

\section{Metodologia}

Trata-se de um estudo exploratório, descritivo de abordagem qualitativa realizada na Pró-Reitoria de Extensão (PROEXTI) de uma universidade publica federal do Amazonas. A amostra consistiu do universo de relatórios finais dos projetos de extensão na modalidade de Ação Curricular de Extensão (ACE) de 2016 e 2017 dos cursos de Educação Física, Enfermagem, Farmácia, Fisioterapia, Medicina e Odontologia. Da amostra inicial de 95 relatórios identificados, 26 foram excluídos por estarem incompletos. A amostra final consistiu em 69 relatórios finais, sendo 27 relatórios do primeiro semestre de 2016 e 26 do segundo semestre. Em 2017 foram encontrados 7 relatórios no primeiro semestre e 8 no segundo.

A coleta de dados foi realizada mediante a utilização de um instrumento contendo questões relacionadas à caracterização do projeto e atividades que contemplavam políticas públicas e educação em saúde. O instrumento possibilitou a obtenção de informações como título da ACE, quantidade de pessoas atendidas, articulação com ensino e/ou pesquisa, instituições parceiras como unidades de saúde ou instituições de ensino, atividades de educação em saúde e metodologias adotadas para a explanação do conteúdo, desenvolvimento de atividades de saúde para a população indígena ou ribeirinha, comunidade atendida e a avaliação da comunidade por meio dos relatos, e para manter o sigilo, os relatos foram identificados com a letra $F$ seguidos de número.

A análise de conteúdo de Bardin (2011) embasou o tratamento dos dados coletados, a qual foi realizada de forma sistemática e criteriosa, em busca de desvelar os significados contidos nos documentos. No primeiro momento os dados coletados foram explorados e organizados por meio de leitura para levantamento quantitativo (quais os assuntos mais presentes) e qualitativo do material baseados no objetivo da pesquisa, bem como inicio da codificação do material com vistas à interpretação e obtenção dos significados. Posteriormente realizou-se leitura exaustiva do material e da literatura para sistematização das ideias, recortes, classificação e enumeração do material para a construção das unidades de análise. Nessa fase, fez-se necessário interpretar o material coletado para a elaboração das categorias. No terceiro momento deu-se o tratamento dos resultados obtidos 
e a interpretação. Nessa fase, houve necessidades de leitura do material e da literatura sobre o assunto com o intuito identificar temas ainda não incluídos e realizar alterações para interpretação a luz da literatura.

Por tratar-se de uma pesquisa documental, não foi submetido ao Comitê de Ética em Pesquisa.

\section{Resultados e Discussão}

Para melhor compreensão, realizou-se a caracterização dos projetos de extensão desenvolvidos pelos cursos da área da saúde.

\section{Projetos de extensão desenvolvidos pelos cursos da saúde}

Em relação ao conteúdo das ACEs, pode-se apontar como significativa a abordagem na saúde da mulher com foco no período gravídico, observando-se ausência de projetos sobre a mulher em outros contextos. Questões relevantes, como o aumento dos casos de violência contra mulher, não foram explorados, apesar de em 2017 terem sido registrados 4.473 homicídios dolosos de mulheres, um aumento de 6,5\% em relação a 2016, enquanto o número de estupros no Brasil cresceu 8,4\% de 2016 a 2017, passando de 54.968 para 60.018 casos registrados (AMARANTE, 2019). Daí a importância das ACEs acompanharem de perto a evolução de problemas sociais.

Além disso, foram executadas ACEs voltadas ao enfrentamento do câncer, saúde bucal e ao desenvolvimento motor. Na saúde do idoso as ações compreenderam o autocuidado e os aspectos biopsicossociais. Ações de humanização em saúde também foram realizadas por meio de musicoterapia no hospital. Outras ACEs incluíram dispensação de medicamentos e atividades educativas voltadas à prevenção de intoxicações no contexto escolar. Destaca-se a presença de projetos que levam a prática esportiva e dança para a comunidade.

\section{As Políticas Públicas nas Ações Extensionistas da UFAM}

Evidenciou-se que as políticas públicas mais presentes nas ACEs foram: Política Nacional de Atenção Integral à Saúde da Criança com 13 projetos, Política Nacional de Saúde da Pessoa Idosa com 10 e Política Nacional de Atenção Integral à Saúde da Mulher (PNAISM) com seis.

Não foram identificados relatórios que abordassem às seguintes políticas públicas: Política Nacional para a População em Situação de Rua; Atenção Integral à Saúde de Adolescentes; Saúde Integral das Populações do Campo, da Floresta e das Águas; Saúde Integral de Lésbicas, Gays, Bissexuais, Travestis e Transexuais; Atenção Integral em Reprodução Humana Assistida; Atenção Integral às Pessoas com Doenças Raras; Saúde Integral da População Negra; Atenção ao Portador de Doença Renal; Atenção Integral às Pessoas com Doença Falciforme e outras Hemoglobinopatias e Atenção Integral em Genética Clínica.

A ausência de tais políticas públicas nas ACEs pode estar relacionada a dificuldades estruturais que prejudicam a implantação e implementação das mesmas em nosso contexto. O desenvolvimento de ações relacionadas às políticas públicas requer que profissionais estabeleçam parcerias para promover a resolução de problemas, por meio da discussão e causalidade, sem deixar de considerar o conflito de interesses, a ética e o desenvolvimento de negociação para a tomada de decisão (Brasil, 2015).

Conforme Oliveira (2018), a relação existente entre as políticas públicas, vulnerabilidade, sofrimento do ser humano e negligência de pessoas e territórios merece ser discutida e melhor entendida. Uma das principais estratégias é a aposta em diálogos para a produção de conhecimentos sobre saúde em diferentes quadros de referência, como na visão biomédica, determinação social, vulnerabilidade social e aportes descoloniais. 


\section{A Educação em Saúde nas ações extensionistas da UFAM}

Em relação às atividades que contemplavam educação em saúde, no primeiro semestre de 2016, dos 27 relatórios analisados, 14 utilizaram essa metodologia como recurso para promover políticas públicas de saúde. Desses, 6 da enfermagem, um da odontologia, 6 da educação física e fisioterapia e um da farmácia. No segundo semestre, foram 19 projetos, sendo 11 de educação física e fisioterapia, 7 da enfermagem, quatro de odontologia e um de farmácia.

No primeiro semestre de 2017 foram identificadas 6 ACEs, destas três do curso de odontologia, dois da enfermagem e um da farmácia. No segundo semestre foram 8 , tres da enfermagem, dois da educação física e fisioterapia, um da odontologia e um da farmácia. As metodologias de ensino utilizadas compreenderam palestras, cartazes informativos, álbuns seriados, rodas de conversa, vídeos educativos, jogos e teatro.

Quanto ao foco das ações promovidas pelas unidades acadêmicas, observaram-se divergências entre os cursos, o que pode estar relacionado ao perfil da profissão, que pode ter o foco na promoção da saúde ou tratamento de doenças. Pode-se apontar que nas ações dos cursos de educação física e fisioterapia, a promoção à saúde é realizada de forma recorrente haja vista que as ações envolvem atividades físicas e dinâmicas de grupo, além da educação em saúde ser utilizada como ferramenta de motivação para as mudanças de hábitos na comunidade alcançada pelas ACEs.

No curso de enfermagem, predominaram ações voltadas à saúde da criança, da mulher no contexto da obstetrícia e também à saúde do idoso, através de atividades de humanização em saúde que também se apropriam da educação em saúde como forma de cuidado.

No curso de odontologia, a promoção da saúde bucal foi o tipo de atividade mais recorrente, na qual os voluntários envolvidos atuavam tanto no contexto da comunidade promovendo educação em saúde como no contexto ambulatorial realizando atendimento clínico.

$\mathrm{Na}$ medicina foram realizadas atividades de educação em saúde em suas ações extensionistas, no entanto, houve a predominância de ações de extensão no âmbito ambulatorial, com maior recorrência para ações voltadas à saúde da mulher. Ressalta-se que foi a única unidade a promover um projeto para mulheres privadas de liberdade.

No curso de farmácia, as ações mais recorrentes eram voltadas às questões laboratoriais, relacionadas ao perfil do curso voltado à pesquisa. As ações desenvolvidas estavam relacionadas à verificação da adesão, manipulação e dispensação de medicamentos. A farmácia foi o curso com menos atividades de extensão, das quais não foram identificadas educação em saúde.

A análise dos relatórios finais permitiu a compreensão do desenvolvimento das atividades de extensão e a construção de três categorias que melhor representam a interpretação das atividades de extensão executadas e as relações entre políticas públicas e educação em saúde, bem como seus impactos na comunidade alvo.

\section{A extensão como ferramenta para aperfeiçoamento do aprendizado da comunidade e do discente}

Abordou-se a importância das ações de extensão para a ampliação de informações e conhecimento para o público alvo e o impacto no aprendizado é visto não apenas na comunidade assistida, mas também nos discentes envolvidos na extensão.

\footnotetext{
"Eu agora quando for para a maternidade ter meu filho, não irei deixar que me façam nenhum tipo de violência, pois agora eu sei quais os meus direitos como gestante" F6

“Aprendi muito, muito mesmo! Todas as atividades foram de grande importância!” F8
}

Nesse sentindo, pode-se observar o quanto o aprendizado adquirido durante as atividades foi marcante para os envolvidos. Para Santos et al. (2014), pode parecer que, em um primeiro instante, a extensão tenha o intuito de fornecer 
informação à comunidade, porém ao deparar-se com um contexto social enriquecido de diferentes tipos de saberes, é desvelada a "via de mão dupla" para a organização desses saberes.

Segundo Fraga (2017), a transferência de saberes sempre esteve na relação da universidade para com a sociedade. A ideia de que a universidade deve oferecer às classes populares o conhecimento que é produzido está na origem da palavra extensão e se permanece presente durante toda sua jornada histórica.

Para Santos et al. (2014), a extensão universitária precisa estar associada às políticas públicas e articulada ao Projeto Pedagógico de Curso das universidades, uma vez que a indissociabilidade entre ensino, pesquisa e extensão deve estar alicerçada ao perfil profissional e cidadão que se deseja formar.

Conforme Silva (2017), a inserção do discente de graduação em atividades de pesquisa e extensão de forma regular, fornece o entendimento dos Determinantes Sociais de Saúde (DSS) na realidade local. Esse conhecimento construído em conjunto com o professor, sendo valorizada a experiência individual de cada aluno, sua história de vida, seus conhecimentos prévios e o conteúdo novo apresentado permanecerá em seu cognitivo para a aplicação quando profissional.

O desenvolvimento de ações extensionistas, a partir das atividades práticas, oferece aos discentes a oportunidade de experimentar diferentes sentimentos. A troca de saberes entre docentes e discentes corrobora para a construção de experiências significativas por meio dos pensamentos, sentimentos e ações. Tudo aquilo que é apreendido nesses momentos durante a graduação assume um significado para o discente e contribui para que o exercício profissional seja realizado com autonomia no ponto de vista de transformação social (SILVA, 2017).

\section{A extensão como ferramenta para mudança de hábitos de vida da comunidade}

Observaram-se relatos relacionados à mudança nos hábitos de vida com impacto positivo para as pessoas que participaram das ações de extensão. A seguir, foram elencados relatos pertinentes referentes ao conteúdo.

\footnotetext{
"Houve melhorias na escola, no convivio familiar e entre amigos" F11

"Ajudou na perda de peso" F12

"O projeto me ajudou a sair da depressão, estava sem esperança" F10

"Hoje tenho minha vida renovada através desse projeto" F14

"Me faz sentir bem a atividade fisica" F15
}

Pode-se inferir que as atividades trouxeram melhorias na saúde dos envolvidos por meio do aprendizado, o que foi possível identificar nos relatos da comunidade, os quais descreviam a satisfação em ser alvo das atividades.

Os benefícios das atividades extensionistas relacionam-se também ao desenvolvimento na vida de cada ser que ocorrem por meio do compartilhamento do conhecimento, provocando assim, mudanças sociais nos que estão na condição do aprender, o que os leva a contribuírem para a construção de um mundo melhor (Rodrigues e Santos, 2013).

De acordo com Pereira (2013), a participação das universidades em programas públicos é necessária para estimular a consciência crítica por parte da população e para promover o crescimento de seu poder de reivindicação. Logo, o caminho mais adequado para contribuir com a erradicação dos problemas mais complexos da sociedade é incentivar maior interação entre a universidade com as camadas sociais mais pobres.

Segundo Silva, Ribeiro e Júnior (2013), a potencialidade extensionista na formação em saúde é capaz de produzir cuidado integral, desde que haja articulação acadêmica e se concilie assistência à indissociabilidade ensino-pesquisa-extensão; extensão é o espaço de vivências e confrontos entre teoria e prática numa dinâmica dialógica, multiprofissional e socialmente 
comprometida.

Conforme Scharf et al. (2016), as atividades que ultrapassam os muros da universidade possibilitam a construção de novos conhecimentos, fazendo com que os envolvidos adquiram uma visão mais desenvolvida do mundo em que vivem.

\title{
Impacto das Ações de Extensão na comunidade
}

Nessa categoria foram elencadas descrições pertinentes de pessoas alcançadas pelas ações de extensão, as quais se utilizavam de estratégias de cuidado voltadas à humanização, o que evidencia que o mesmo era visto em suas particularidades e atendido com qualidade. As mesmas foram apontadas abaixo:

\author{
"Ver o sorriso do meu filho foi bastante gratificante" F3 \\ "Melhora o sorriso e a condição psicológica" F4 \\ "Sou muito grato pela atenção e carinho que recebi por parte de toda equipe" F5
}

Desse modo, as atividades de extensão trouxeram impactos positivos à população alvo das atividades extensionistas no âmbito da saúde. De acordo com Divino et al. (2013), a extensão universitária possui importância indispensável na forma em que se integra à rede assistencial e pode atuar como espaço para vivência de novas experiências voltadas à humanização, ao cuidado e à qualificação da atenção a saúde.

Nota-se que a graduação na área da saúde agregada a extensão universitária mostra-se fundamental para a vivência de experiências na atuação em diversos cenários do trabalho em saúde. O desenvolvimento de projetos de extensão na área da saúde denota notável importância para a população assistida (Biscarde, Pereira-Santos e Silva, 2014).

Portanto, é necessário dimensionar a extensão como uma parte integrante da formação, que não é menos importante que a pesquisa ou ensino, de maneira a compreender e refletir em suas práticas o seu modo social, político e científico (Ribeiro et al., 2016).

Observa-se que a extensão desenvolvida na universidade tem potencial para alcançar a comunidade e transformá-la, por ser um espaço de compartilhamento dos conhecimentos produzidos na universidade, de construção de conhecimentos junto à comunidade em busca de resolução de problemas que a aflige, considerando o modo de vida, o contexto político, social e cultural da população assistida. Para Albrecht e Bastos, 2020), a extensão oportuniza o diálogo, a desconstrução das certezas e dogmas que mantem os modelos existentes, e o compartilhamento do conhecimento constrói uma sociedade inclusiva.

\section{Conclusão}

O estudo proporcionou o conhecimento da extensão universitária dos cursos de saúde de uma universidade do Amazonas e as relações desta com as políticas públicas e educação em saúde. O estudo permitiu caracterizar as ACEs dos cursos e evidenciou que os mesmos possuem em sua essência atividades que contemplam políticas públicas de saúde.

Observou-se o imponente impacto das atividades de extensão na qualidade de vida da população atendida. Contudo, ainda existe a necessidade de desenvolver projetos relacionados às políticas voltadas às pessoas em situação de rua, em conflito com a lei, às lésbicas, gays, bissexuais, travestis e transexuais, à população negra, do campo, da floresta e das águas, assim como as pessoas com doenças raras. Visto que estas políticas não foram contempladas nas atividades no período estudado.

As atividades de extensão desenvolvidas nos cursos da saúde foram significativas para a comunidade, pois foi identificado impacto positivo na saúde por meio do aprendizado e da transformação de hábitos de vida, além da promoção da saúde e prevenção de agravos à saúde, refletindo em benefícios para a comunidade atendida. Ademais, a extensão é importante e 
Research, Society and Development, v. 10, n. 4, e58010414379, 2021

(CC BY 4.0) | ISSN 2525-3409 | DOI: http://dx.doi.org/10.33448/rsd-v10i4.14379

necessária para a formação do futuro profissional.

Contudo, é necessário incentivar a criação e execução de novos projetos de extensão para que a universidade cumpra seu papel na transformação social, promovendo saúde para populações mais vulneráveis com vistas a reduzir as iniquidades encontradas em nossa realidade.

\section{Referências}

Albrecht, E., \& Bastos, A. S. A. M. (2020). Extensão e sociedade: diálogos necessários. Revista Em Extensão 19(1), 54-71. Uberlandia Evonir Albrecht, Antonio Sergio Abrahão Monteiro Bastos.

Amarante, S. (2019). Violência contra as mulheres vem crescendo no Brasil. IFF/FIOCRUZ. https://portal.fiocruz.br/noticia/violencia-contra-mulheres-vemcrescendo-no-brasil.

Bardin, L. (2011). Análise de Conteúdo (70ª ed.): LDA.

Biscarde, D. G. S., \& Pereira-Santos, M. \& Silva, L. B. (2014). Formação em saúde, extensão universitária e Sistema Único de Saúde (SUS): conexões necessárias entre conhecimento e intervenção centradas na realidade e repercussões no processo formativo. Comunicação saúde educação. 18(48): 177-86 10.1590/1807-57622013.0586. https://www.scielo.br/pdf/icse/v18n48/1807-5762-icse-18-48-0177.pdf

Capella, A. C. N. Formulação de Políticas. (2018): Enap,. 151 p.: il. ISBN: 978-85-256-0080-6.

Divino, A. E. A., Oliveira, C. E. L., Costa, C. A. C., Neta, H. R. S., Campos, L. S., Menezes, R. M. J., Cabral, S. C. S., \& Costa, C. L. N. A. (2013). A extensão universitária quebrando barreiras. Cadernos de Graduação - Ciências Humanas e Sociais. 1(16), 135-140.

Fraga, L. S. (2017). Transferência de conhecimento e suas armadilhas na extensão universitária brasileira. Avaliação. 22(2), 403-419.

Medeiros, M. M. (2017). A extensão universitária no Brasil - um percurso histórico. Revista Barbaquá/UEMS. 01(01), 09-16.

Ministério da Saúde. (2015). Curso de aperfeiçoamento: implementação da Política de Promoção da Saúde: Programa Academia da Saúde. Universidade Federal de Santa Catarina. - Brasília.

Minayo, M. C. (2014). Análise qualitativa: teoria, passos e fidedignidade. Ciência \& amp; Saúde Coletiva. 17(3), 621-626.

Oliveira, R. G. (2018). Práticas de saúde em contextos de vulnerabilização e negligência de doenças, sujeitos e territórios: potencialidades e contradições na atenção à saúde de pessoas em situação de rua. Saúde Soc. São Paulo, 27(1), 37-50.

Perera, L. B. (2013). Extensão universitária e políticas públicas. Revista Extensão \&amp; Cidadania. 1(1), 91-104.

Ribeiro, M. A., Cavalvante, A. S. P., Albuquerque, I. M. N., Vasconcelos, M. I. O. (2016). A extensão universitária na perspectiva de estudantes de cursos de graduação da área da saúde. Interagir: pensando a extensão. 2236-4447 (21), 55-69.

Rios, D. R. S \& Caputo, M. C. (2019). Para Além da Formação Tradicional em Saúde: Experiência de Educação Popular em Saúde na Formação Médica. Rev. bras. educ.med. 43(3), 184-195.

Rodrigues, A. L. L., Prata, M. S., Batalha, T. B. S., Costa, C. L. N. A \& Neto, I. F. P. (2013). Contribuições da Extensão Universitária na Universidade. Cadernos de Graduação - Ciências Humanas e Sociais. 1(16), 141-148.

Salci, M. A. (2013). Educação em saúde e suas perspectivas teóricas: algumas reflexões. Texto Contexto Enferm. Florianópolis.

Santos, A. B., Abib, S. W., Santos, V. M. M., Souza, S. S., \& Gomes, N. P. (2014). Extensão universitária: a visão de acadêmicos da Universidade do Estado de Santa Catarina (UDESC). Em Extensão. 12(2), 9-22, 3.

Scharf, D., Oliveira, M. F., Oliveira, A., Schlindwein, C. A., Rastelli, M. C. S., \& Andrade, I. C. G. B. (2016). Odontologia itinerante na extensão universitária: FURB Móvel - promovendo saúde bucal e cidadania. Revista Brasileira de Extensão Universitária. 7(1), 37-42.

Silva, A. F. L., Ribeiro, C. D. M., Júnior, A. G. S. et al. (2013). Pensando extensão universitária como campo de formação em saúde: uma experiência na Universidade Federal Fluminense, Brasil. Interface (Botucatu). 17(45), 371-384.

Silva, M. B. T. (2017). Inserção do acadêmico de Enfermagem em atividades de pesquisa e extensão universitária: uma estratégia de ensino. Tese (Doutorado) Instituto Oswaldo Cruz, Pós-Graduação em Ensino em Biociências e Saúde, Rio de Janeiro, Rio de Janeiro, Brasil.

Sousa, A. J., Carneiro, S. R. O., \& Rocha, V. O. Extensão universitária na UFRB. (2017). UFRB. 